\title{
Uniqueness, stability and numerical reconstruction of a time and space-dependent conductivity for an inverse hyperbolic problem
}

\author{
L. Beilina, M. Cristofol and S. Li
}

This paper is devoted to the reconstruction of the time and space-dependent coefficient in an inverse hyperbolic problem in a bounded domain. Using a local Carleman estimate we prove the uniqueness and a Hölder stability in the determining of the conductivity by a single measurement on the lateral boundary. Our numerical examples show possibility of the determination of the location and the large contrast of the space-dependent function in three dimensions.

Key words: Inverse Problem, Carleman estimate, time and space-dependent coefficient, infinite domain, hyperbolic equation

\section{Introduction}

Result of this paper is based on two recent works [2, 12]. In [12] the authors deal with the inverse problem of determining the time-independent isotropic conductivity coefficient $c: \Omega \rightarrow \mathbb{R}$ appearing in the hyperbolic partial differential equation $\left(\partial_{t}^{2}-\right.$ $\nabla \cdot \tilde{c} \nabla) u=0$, where $\Omega:=\omega \times \mathbb{R}$ is an infinite cylindrical domain whose cross section $\omega$ is a bounded open subset of $\mathbb{R}^{n-1}, n \geq 2$. On the other hand, in [2] the authors

\footnotetext{
L. Beilina

Department of Mathematical Sciences, Chalmers University of Technology and University of Gothenburg, SE-41296 Gothenburg Sweden, e-mail: larisa.beilina@chalmers.se

M. Cristofol

Institut de Mathématiques de Marseille, CNRS, UMR 7373, École Centrale, Aix-Marseille Université, 13453 Marseille, France,

S. Li

Key Laboratory of Wu Wen-Tsun Mathematics, Chinese Academy of Sciences, School of Mathematical Sciences, University of Science and Technology of China, 96 Jinzhai Road, Hefei, Anhui Province, 230026, China
} 
extend the result of [12] to a more general class of conductivities: time and spacedependent conductivities $\tilde{c}(x, t)$.

The goal of this work is to modify the uniqueness and stability result of [2] for the case of bounded domain $\Omega$ and a finite number of observations. Indeed, in the case of infinite cylindrical domain, some strong assumptions are required concerning the coefficient to be reconstructed (see condition (2.3) in [2]). In the current work we weaken these conditions for the case of a bounded domain what will lead to the new stability estimate. Furthermore, in a new stability inequality we have derived estimate of the distance of two sets of coefficients $\tilde{c}_{1}(x, t)$ and $\tilde{c}_{2}(x, t)$ through the distance of observations at the lateral boundary of the Neumann derivative of the solutions $u_{1}$ and $u_{2}$, respectively. This stability inequality implies the uniqueness of the determination of the coefficient $\tilde{c}$. Furthermore, we can use it in numerical studies of the determination of the space and time-dependent function $\tilde{c}(x, t)$ from the backscattered noisy observations.

We consider the following initial boundary value problem

$$
\begin{cases}\partial_{t}^{2} u-\operatorname{div}(\tilde{c}(x, t) \nabla u(x, t))=0 & \text { in } Q:=\Omega \times(0, T), \\ u(\cdot, 0)=a(x), \partial_{t} u(\cdot, 0)=0 & \text { in } \Omega, \\ u=0 & \text { on } \Sigma:=\partial \Omega \times(0, T),\end{cases}
$$

where $\Omega$ is a bounded domain and with the set of initial conditions $(a, 0)$, where $\tilde{c}$ is the unknown conductivity coefficient. We assume that $\tilde{c}$ is time and space depending conductivity such that:

$$
\tilde{c}(x, t)=c_{0}(x, t)+c(x),
$$

where $c_{0}(x, t)$ is assumed to be known. In other words, we consider the case of the perturbation of a general time and space-dependent conductivity $\tilde{c}(x, t)$ by a spacedependent one $c(x)$. In [21] the authors study a similar general non-stationary media for an inverse problem for Maxwell's equations.

The derivation of the stability and uniqueness result of this work is based on a Carleman inequality specifically designed for hyperbolic systems, see, for example, $[7,8,9,10,11,15,16,18,19,20,22]$ where have been proven stability results of the reconstruction of one or several unknown coefficients in a hyperbolic equation from a finite number of measurements. However, only theoretical investigations are presented in all these works. Theoretical uniqueness results together with numerical simulations are presented in recent works $[2,3]$.

In numerical examples of this work the goal was to determine the conductivity function $\tilde{c}(x, t)$ that represents a sum of two space-dependent gaussians and one time-dependent function. Since by our assumption the time-dependent function $c_{0}(x, t)$ is known inside the domain, then we have reconstructed only the spacedependent part of the conductivity function, $c(x)$. To do that we used the Lagrangian approach together with the domain decomposition finite element/finite difference method of [5]. One of the important points of this work is that in our numerical simulations we applied one non-zero initial condition in the model problem which corresponds well to the uniqueness and stability results of this paper. Our threedimensional examples show that we can determine the location and the large con- 
trast of the space-dependent function. However, the location of this function in the third, $x_{3}$ direction, should be improved. This can be done using an adaptive finite element method, see details in $[3,4,6]$.

The outline of the work is the following: in section 2 we derive the main stability estimate and the uniqueness result, in section 3 we present numerical simulations and in section 4 we give conclusions to the work.

\section{Main theoretical result}

\subsection{Settings and main result}

Let $\Omega \subset \mathbb{R}^{n}$ be a bounded domain with smooth boundary $\partial \Omega$. We consider an acoustic equation

$$
\partial_{t}^{2} u(x, t)-\operatorname{div}(\tilde{c}(x, t) \nabla u(x, t))=0, \quad x \in \Omega, 0<t<T .
$$

To (1) we attach the initial and boundary conditions:

$$
u(x, 0)=a(x), \quad \partial_{t} u(x, 0)=0, \quad x \in \Omega
$$

and

$$
u(x, t)=h(x, t), \quad(x, t) \in \partial \Omega \times(0, T) .
$$

Throughout this paper, we set $\partial_{j}=\frac{\partial}{\partial x_{j}}, \partial_{i} \partial_{j}=\frac{\partial^{2}}{\partial x_{i} \partial x_{j}}, \partial_{t}^{2}=\frac{\partial^{2}}{\partial t^{2}}, 1 \leq i, j \leq n$.

Let $T>0$ be given. We assume that coefficient $\tilde{c}=\tilde{c}(x, t)$ of the principal term of (1) is time and space depending in form (2) where $c_{0}(x, t)$ is given. In this paper, we consider an inverse problem of determining time-independent component $c(x)$ of coefficient $\tilde{c}(x, t)$, from the observations:

$$
u(x, t), \quad x \in \partial \Omega \times(0, T)
$$

In order to formulate our results, we need to introduce some notations. Let $x_{0} \notin \bar{\Omega}$ and $\beta>0$. We set

$$
\Lambda=\left(\sup _{x \in \Omega}\left|x-x_{0}\right|^{2}-\inf _{x \in \Omega}\left|x-x_{0}\right|^{2}\right)^{\frac{1}{2}} .
$$

We define admissible sets of conductivity coefficients. For arbitrarily fixed functions $\eta_{0} \in C^{2}(\bar{\Omega} \times[0, T]), \eta_{1} \in\left(C^{2}(\bar{\Omega} \times[0, T])\right)^{n}$ and constants $M_{1}>0, \theta_{0}>0, \theta_{1}>0$, we set

$\mathscr{U}=\mathscr{U}_{M_{1}, \theta_{1}, \eta_{0}, \eta_{1}, c_{0}}=\left\{\tilde{c}(x, t)=c_{0}(x, t)+c(x) \in C^{2}(\bar{\Omega} \times[0, T]) ; \tilde{c}=\eta_{0}, \nabla \tilde{c}=\eta_{1}\right.$ on $\partial \Omega \times[0, T]$, 
$\|\tilde{c}\|_{C^{2}(\bar{\Omega} \times[0, T])} \leq M_{1}, \tilde{c}(x, t) \geq \theta_{1}, \frac{\left(x-x_{0}\right) \cdot \nabla \tilde{c}(x, t)}{2 \tilde{c}(x, t)}<1-\theta_{0}$ for all $\left.(x, t) \in \bar{\Omega} \times[0, T]\right\}$.

We note that there exists a constant $M_{0}>0$ such that $\left\|\nabla_{x, t} \tilde{c}\right\|_{C(\bar{\Omega} \times[0, T])} \leq M_{0}$ for each $\tilde{c} \in \mathscr{U}_{k}, k=1,2$.

By $x_{0} \notin \bar{\Omega}$, there exists a constant $\beta>0$ such that

$$
\beta+\left\{\frac{1}{2 \theta_{1}}+\frac{1}{\sqrt{\theta_{1}}}\right\} \lambda M_{0} \sqrt{\beta}<\theta_{1} \theta_{0}, \quad \theta_{1} \inf _{x \in \Omega}\left|x-x_{0}\right|^{2}>\beta \Lambda^{2} .
$$

We point out that, if $c_{0}(x, t)$ satisfies $\frac{t \partial_{t} c_{0}(x, t)}{c_{0}(x, t)} \geq 0$ for all $(x, t) \in \bar{\Omega} \times[0, T]$, the first inequality in (6) can be replaced with

$$
\beta+\frac{\lambda M_{0}}{\sqrt{\theta_{1}}} \sqrt{\beta}<\theta_{1} \theta_{0}
$$

We are ready to state our first main result.

Theorem 1. We fix $a \in C^{2}(\bar{\Omega})$ satisfying

$$
\left(\nabla a(x) \cdot\left(x-x_{0}\right)\right)>0 \quad \text { for all } x \in \bar{\Omega}
$$

Assume that $c_{0} \in W^{4, \infty}(Q ; \mathbb{R})$ and $\partial_{t} c_{0}(\cdot, 0)=\partial_{t}^{3} c_{0}(\cdot, 0)=0$ in $\Omega$. Let $\mathscr{U}$ be defined by (5) and let $\beta>0$ satisfy (6). We assume that

$$
T>\frac{\Lambda}{\sqrt{\beta}} .
$$

We pick arbitrarily $\tilde{c}_{1}, \tilde{c}_{2} \in \mathscr{U}$ such that the solutions $u_{j}, j=1,2$ to (1)-(3) where $\tilde{c}_{j}$ is substituted to $\tilde{c}$ are in $W^{4, \infty}(\Omega \times(0, T)$. Then there exists a constant $C>0$ such that

$$
\left\|\tilde{c}_{1}-\tilde{c}_{2}\right\|_{H^{1}(\Omega)} \leq C\left\|\frac{\partial u_{1}}{\partial v}-\frac{\partial u_{2}}{\partial v}\right\|_{H^{3}\left(0, T ; L^{2}(\partial \Omega)\right)}^{\kappa},
$$

where $v=v(x)$ denotes the unit outward normal vector to $\partial \Omega$ at $x$. Here $C>0$ and $\kappa \in(0,1)$ are two constants depending only on $\Omega, T, x_{0}, M_{1}, \theta_{1}, \eta_{0}, \eta_{1}, a(x)$ and $\left\|u_{j}\right\|_{W^{4, \infty}(\Omega \times(0, T)}, j=1,2$.

\subsection{The Carleman estimate for a hyperbolic equation}

We consider a Carleman estimate which is derived from [17].

Let us set

$$
Q=\Omega \times(-T, T) .
$$


For $x_{0} \notin \bar{\Omega}$ and $\beta>0$, we define the functions $\psi=\psi(x, t)$ and $\varphi=\varphi(x, t)$ by

$$
\psi(x, t)=\left|x-x_{0}\right|^{2}-\beta t^{2}
$$

and

$$
\varphi(x, t)=e^{\lambda \psi(x, t)}
$$

with parameter $\lambda>0$. We add a constant $C_{0}>0$ if necessary so that we can assume that $\psi(x, t) \geq 0$ for $(x, t) \in Q$, so that

$$
\varphi(x, t) \geq 1, \quad(x, t) \in \bar{Q} .
$$

Lemma 1. [17] We assume $\mu \in C^{1}(\bar{Q})$, and satisfies the following conditions

$$
\frac{\beta}{\mu}\left[1-\frac{t \partial_{t} \mu}{2 \mu}+\frac{|t||\nabla \mu|}{\sqrt{\mu}}\right]<1-\frac{\left(x-x_{0}\right) \cdot \nabla \mu}{2 \mu} \quad \text { for all } \quad(x, t) \in \bar{Q}
$$

and

$$
\mu(x, t)\left|x-x_{0}\right|^{2}-\beta^{2} t^{2}>0 \quad \text { for all } \quad(x, t) \in \bar{Q}
$$

even by choosing $\beta>0$ sufficiently small. Let $y \in H^{1}(Q)$ satisfy

$$
\partial_{t}^{2} y(x, t)-\mu(x, t) \Delta y=F \quad \text { in } Q
$$

and

$$
y(x, t)=0, \quad(x, t) \in \partial \Omega \times(-T, T), \quad \partial_{t}^{k} y(x, \pm T)=0, \quad x \in \Omega, k=0,1 .
$$

We fix $\lambda>0$ sufficiently large. Then there exist constants $s_{0}>0$ and $C>0$ such that

$$
\int_{Q}\left(s\left|\nabla_{x, t} y\right|^{2}+s^{3}|y|^{2}\right) e^{2 \tau \varphi} d x d t \leq C \int_{Q}|F|^{2} e^{2 \tau \varphi} d x d t+C s \int_{-T}^{T} \int_{\partial \Omega}\left|\frac{\partial y}{\partial v}\right|^{2} e^{2 \tau \varphi} d x d t
$$

for all $s>s_{0}$.

Proof. By (11), we can see that $a_{0}(x, t)=1 / \sqrt{\mu(x, t)}$ satisfies (17) in P. 101 of [17]. From (12) it follows that $A\left(x, t ; \nabla_{x, t} \psi(x, t)\right)=\mu(x, t)|\nabla \psi(x, t)|^{2}-\left|\partial_{t} \psi(x, t)\right|^{2}$ is not zero at any point of $\bar{\Omega}$. Therefore, by Theorem 2.1 in [17] and the proof of Corollary 2.5 in [17], we can conclude that, for some sufficiently large $\lambda>0$, there exist constants $s_{0}>0$ and $C>0$ such that (15) holds for all $s>s_{0}$.

Henceforth $C>0$ denotes generic constants which are independent of choices of $\tilde{c}_{1}, \tilde{c}_{2} \in \mathscr{U}$ and the parameter $s>0$ in the Carleman estimates, provided that $s>s_{0}$. 


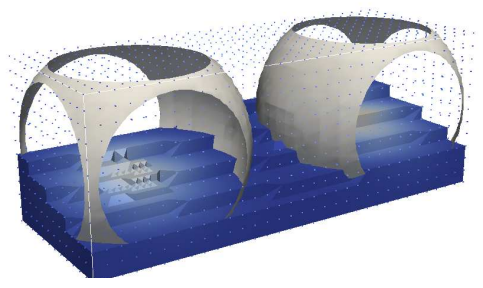

a) $c(x)=1.2$

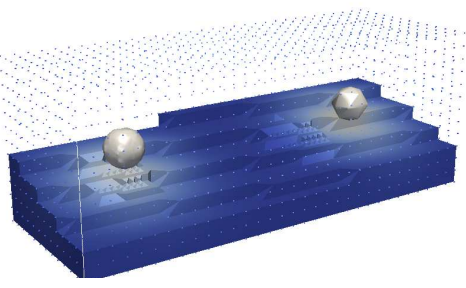

b) $c(x)=5.5$

Fig. 1 Slices of the exact Gaussian function $c(x)$ in $\Omega_{F E M}$ given by (4).

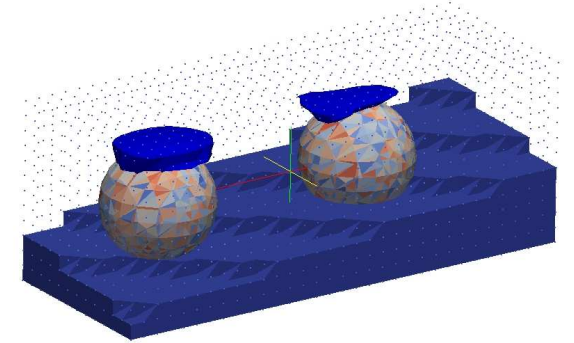

a) $\sigma=3 \%$

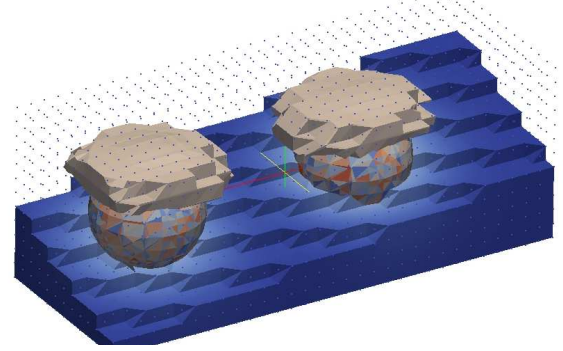

b) $\sigma=10 \%$

Fig. 2 Reconstructions obtained in Test 1 for different noise level $\sigma$ in data. All figures are visualized for $c(x)=3.5$.

\section{Numerical Studies}

This section presents numerical examples of the reconstruction of the unknown function $c(x)$ in the equation (1). To do this we use the domain decomposition method of [5] and decompose $\Omega$ into two sub-regions $\Omega_{F E M}$ and $\Omega_{F D M}$ such that $\Omega=\Omega_{F E M} \cup \Omega_{F D M}$. In $\Omega_{F E M}$ we will use the finite element method (FEM) and in $\Omega_{F D M}$ - the finite difference method (FDM). We decompose also the boundary $\partial \Omega$ of the domain $\Omega$ such that $\partial \Omega=\partial_{1} \Omega \cup \partial_{2} \Omega \cup \partial_{3} \Omega$. Here, $\partial_{1} \Omega$ and $\partial_{2} \Omega$ are the front and back sides of $\Omega$, respectively, and $\partial_{3} \Omega$ denotes the remaining part of the boundary $\partial \Omega$.

We collect time-dependent observations $S_{T}:=\partial_{1} \Omega \times(0, T)$ at the backscattering side $\partial_{1} \Omega$ of $\Omega$. We define $\Omega_{T}:=\Omega \times(0, T), S_{1,1}:=\partial_{1} \Omega \times\left(0, t_{1}\right], S_{1,2}:=\partial_{1} \Omega \times$ $\left(t_{1}, T\right), S_{2}:=\partial_{2} \Omega \times(0, T)$ and $S_{3}:=\partial_{3} \Omega \times(0, T)$. The model problem used in the computations is following: 


$$
\begin{aligned}
\frac{\partial^{2} u}{\partial t^{2}}-\nabla \cdot(\tilde{c} \nabla u) & =0 \text { in } \Omega_{T}, \\
u(x, 0)=\theta_{0}(x), \quad \partial_{t} u(x, 0) & =0 \text { in } \Omega, \\
\partial_{n} u & =f(t) \text { on } S_{1,1}, \\
\partial_{n} u & =-\partial_{t} u \text { on } S_{1,2}, \\
\partial_{n} u & =-\partial_{t} u \text { on } S_{2}, \\
\partial_{n} u & =0 \text { on } S_{3} .
\end{aligned}
$$

In (1) the function $f(t)$ presents a plane wave which is initialized at $\partial_{1} \Omega$ in time $T=[0,3.0]$. We define it as

$$
f(t)= \begin{cases}\sin (\omega t), & \text { if } t \in\left(0, \frac{2 \pi}{\omega}\right), \\ 0, & \text { if } t \geq \frac{2 \pi}{\omega} .\end{cases}
$$

We initialize initial condition $\theta_{0}(x)$ at the backscattered side $\partial_{1} \Omega$ as

$$
u(x, 0)=f_{0}(x)=\left.\mathrm{e}^{-\left(x_{1}^{2}+x_{2}^{2}+x_{3}^{3}\right)} \cdot \cos t\right|_{t=0}=\mathrm{e}^{-\left(x_{1}^{2}+x_{2}^{2}+x_{3}^{3}\right)} .
$$

We assume that the functions $c(x)=1$ and $c_{0}(x, t)=0$ inside $\Omega_{F D M}$. The goal of our numerical tests is to reconstruct a smooth function $c(x)$ only inside $\Omega_{F E M}$ which we define as

$$
\begin{aligned}
c(x) & =1.0+5.0 \cdot \mathrm{e}^{-\left(\left(x_{1}-0.5\right)^{2} / 0.2+x_{2}{ }^{2} / 0.2+x_{3}{ }^{2} / 0.2\right)} \\
& +5.0 \cdot \mathrm{e}^{-\left(\left(x_{1}+1\right)^{2} / 0.2+x_{2}{ }^{2} / 0.2+x_{3}{ }^{2} / 0.2\right)} .
\end{aligned}
$$

We also assume that the function $c_{0}(x, t)$ is known inside $\Omega_{F E M}$, and we define this function as

$$
c_{0}(x, t)=0.01 \cos t \cdot \mathrm{e}^{-\left(x_{1}^{2} / 0.2+x_{2}^{2} / 0.2+x_{3}^{2} / 0.2\right)} .
$$

Figure 1 presents slices of the exact function $c(x)$ given by (4) for $c(x)=1.2$ and $c(x)=5.5$, correspondingly, and Figure 3 presents isosurfaces of the exact function $\tilde{c}$ in the problem (1) for $\tilde{c}=3.5$ at different times. Numerical tests of [5] show that the best reconstruction results for the space-dependent function $c(x)$ and for $c_{0}=0$ in $\Omega$ are obtained for $\omega=40$ in (2), and we take $\omega=40$ in (2) in all our tests.

We introduce dimensionless spatial variables $x^{\prime}=x /(1 \mathrm{~m})$ and define $\Omega_{F E M}$ and $\Omega_{F D M}$ as the following dimensionless computational domains:

$$
\begin{aligned}
\Omega_{F E M} & =\left\{x=\left(x_{1}, x_{2}, x_{3}\right) ; x_{1} \in(-1.6,1.6), x_{2} \in(-0.6,0.6), x_{3} \in(-0.6,0.6)\right\}, \\
\Omega & =\left\{x=\left(x_{1}, x_{2}, x_{3}\right) ; x_{1} \in(-1.8,1.8), x_{2} \in(-0.8,0.8), x_{3} \in(-0.8,0.8)\right\} .
\end{aligned}
$$

We choose the mesh size $h=0.1$ in the overlapping layers between $\Omega_{F E M}$ and $\Omega_{F D M}$ as well as in the computations of the inverse problem. However, we have generated our backscattered data using the several times locally refined mesh inside 


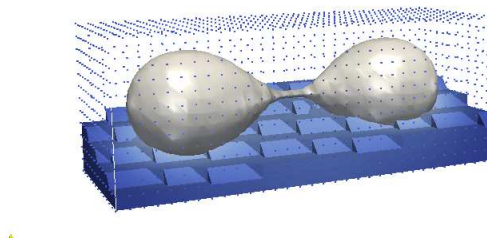

$\mathrm{t}=1.2$

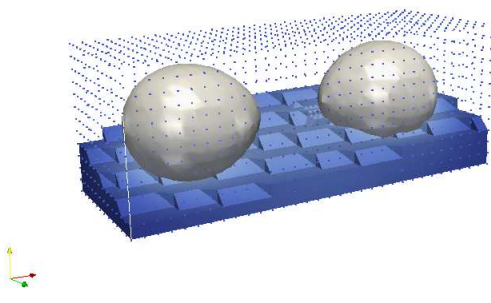

$\mathrm{t}=2.4$

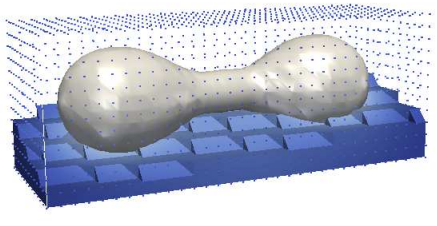

$\mathrm{t}=1.8$

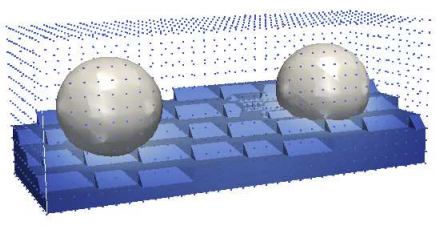

$\mathrm{t}=2.7$

Fig. 3 Slices of the exact space and time-dependent function $\tilde{c}$ for $\tilde{c}=3.5$ at different times.

the domain $\Omega_{F E M}$. To generate backscattered data we solve the model problem (1) in time $T=[0,3.0]$ with the time step $\tau=0.003$ which satisfies the CFL condition [13]. We also use additive noise $\sigma=3 \%, 10 \%$ at $\partial_{1} \Omega$. In all our computations we choose constant regularization parameter $\gamma=0.01$ because it gives smallest relative error in the reconstruction of the function $c(x)$. See $[1,14,23]$ for other techniques of choosing regularization parameter.

We assume that the reconstructed function $c(x)$ belongs to the set of admissible parameters

$$
M_{c} \in\{c \in C(\bar{\Omega}) \mid 1 \leq c(x) \leq 10\}
$$

To get final images of our reconstructed function $c(x)$ we use a post-processing procedure which is the same as in [5]. 
Table 1. Computational results of the reconstructions together with computational errors in the maximal contrast of $c(x)$ in percents. Here, $\bar{N}$ is the final number of iteration in the conjugate gradient method.

\begin{tabular}{|l|l|l|l|l|l|l|l|}
\hline \multicolumn{3}{|c|}{$\sigma=3 \%$} & \multicolumn{4}{c|}{$\sigma=10 \%$} \\
\hline Case & $\max _{\Omega_{\text {IEM }}} c_{\bar{N}}$ & error, $\%$ & $\bar{N}$ & Case & $\max _{\Omega_{F E M}} c_{\bar{N}}$ & error, \% & $\bar{N}$ \\
\hline Test 1 & 6.66 & 11 & 13 & Test 1 & 8.11 & 35 & 15 \\
Test 2 & 4.1 & 32 & 9 & Test 2 & 5.58 & 7 & 10 \\
\hline
\end{tabular}

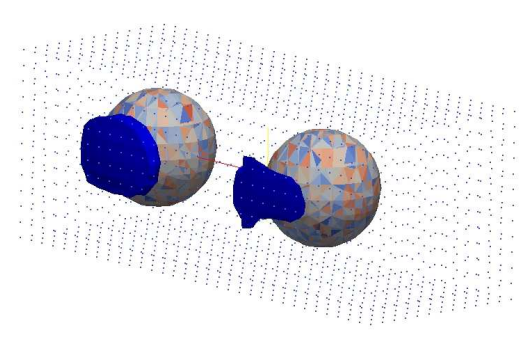

prospect view

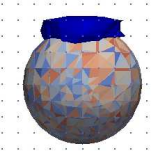

$x_{2} x_{3}$ view

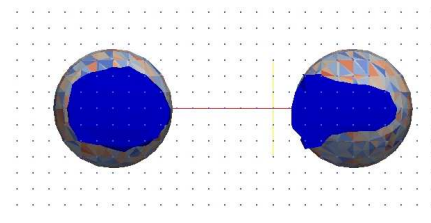

$x_{1} x_{2}$ view

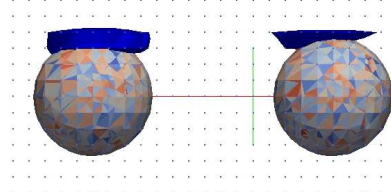

$x_{3} x_{1}$ view

Fig. 4 Test 1. Isosurfaces of the reconstructed function $c(x)$ with $\max _{\Omega_{F E M}} c(x)=6.66$ for $\omega=40$ in (2). Here, the noise level in data is $\sigma=3 \%$. The spherical wireframe of the isosurface with exact value of the function (4), which corresponds to the value of the reconstructed $c=0.7 \max _{\Omega_{F E M}} c(x)$.

\subsection{Test 1}

In this section we present numerical results for determining of the function $c(x)$ given by (4), see Figure 1-a), b), assuming, that the function $c_{0}(x, t)=0$. In this case we obtain results similar to ones of [5].

Figures 4 display results of the reconstruction of the function $c(x)$ given by (4) with additive noise $\sigma=3 \%$ in data. Quite similar results are obtained for $\sigma=10 \%$, see Figure 5. We observe that the location of the maximal value of the function (4) is imaged correctly. It follows from Figure 4 and Table 1 that the imaged contrast in this function is $6.66: 1=\max _{\Omega_{F E M}} c_{13}: 1$, where $n:=\bar{N}=13$ is the final iteration in the conjugate gradient method. Similar observation we made using the Figure 5 and Table 1 where the imaged contrast is $8.11: 1=\max _{\Omega_{F E M}} c_{15}: 1, n:=\bar{N}=15$. These figures show that the values of the background of function (4) are not reconstructed 


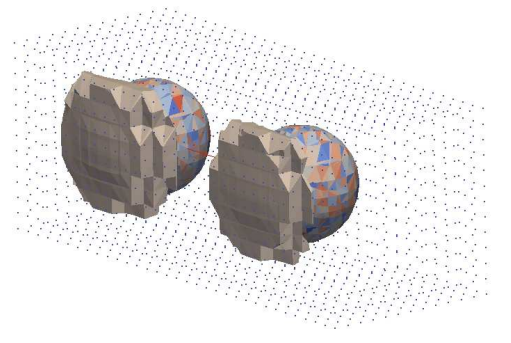

prospect view

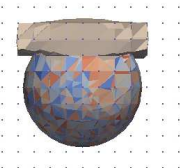

$x_{2} x_{3}$ view

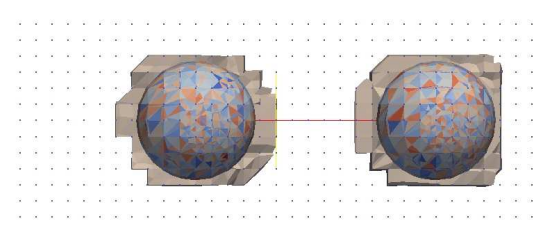

$x_{1} x_{2}$ view

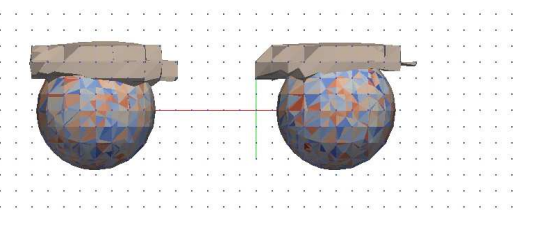

$x_{3} x_{1}$ view

Fig. 5 Test 1. Isosurfaces of the reconstructed function $c(x)$ with $\max _{\Omega_{F E M}} c(x)=8.11$ for $\omega=40$ in (2). Here, the noise level in data is $\sigma=10 \%$. The spherical wireframe of the isosurface with exact value of the function (4), which corresponds to the value of the reconstructed $c=0.7 \max _{\Omega_{F E M}} c(x)$.

but are smoothed out since we are using the data post-processing procedure, see details in $[5,6]$.

Thus, we are able to reconstruct only maximal values of the function (4). Comparison of Figures 1-c), d), 4, 5 with Figure 1-a), b) reveals that it is desirable to improve shape of the function (4) in $x_{3}$ direction.

\subsection{Test 2}

In this numerical test we determine the function $c(x)$ defined in (4) by using noisy backscattered data. To get reasonable reconstruction in this test we run the conjugate gradient algorithm in time $T=[0,1.5]$ with the time step $\tau=0.003$. We note, that we reduced the computational time compared with the first test since by running in a more longer time $T=[0,3.0]$ we have obtained some artifacts at the middle of the domain. From other side, reducing of the computational time was resulted in obtaining of a lower contrast in the reconstructed function, see Table 1 . We tested reconstruction of the function $c(x)$ with the guess values of $c(x)=1.0$ and $c_{0}(x, t)$ as in (5) since by our assumption this function is known. 


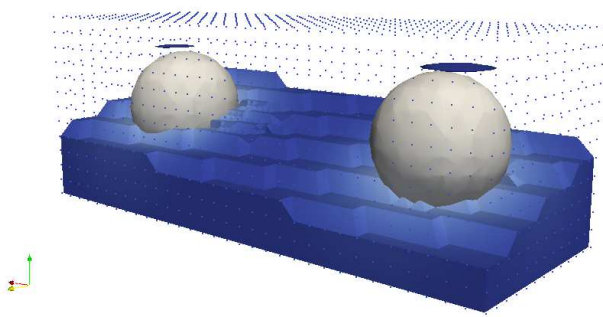

prospect view

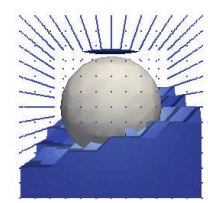

$x_{2} x_{3}$ view

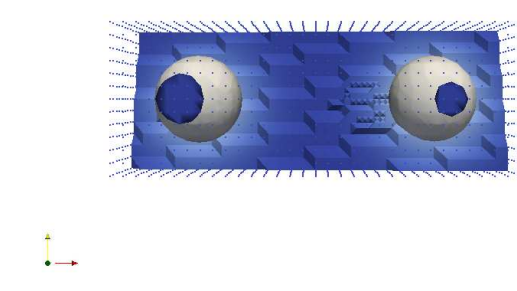

$x_{1} x_{2}$ view

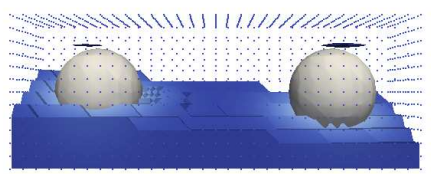

$x_{3} x_{1}$ view

Fig. 6 Test 2. Isosurfaces of the reconstructed function $c(x)$ with $\max _{\Omega_{F E M}} c(x)=4.1$ for $\omega=40$ in (2). Here, the noise level in data is $\sigma=3 \%$. We outline also the spherical wireframe of the isosurface with exact value of the function (4), which corresponds to the value of the reconstructed $c=0.7 \max _{\Omega_{F E M}} c(x)$.

Figures 6, 7 show results of the reconstruction with $\sigma=3 \%$ and $\sigma=10 \%$, respectively. We observe that the location of the maximal value of the function (4) is imaged very well. Again, as in the previous test, the values of the background in (4) are smoothed out. Comparing figures with results of reconstruction we conclude that it is desirable improve shape of the function $c(x)$ in $x_{3}$ direction.

\section{Conclusions}

In this work we presented uniqueness and stability results together with numerical studies of the reconstruction of the time and space-dependent coefficient in an inverse hyperbolic problem in a bounded domain. Using results of previous works $[2,12]$ a local Carleman estimate was proved. 

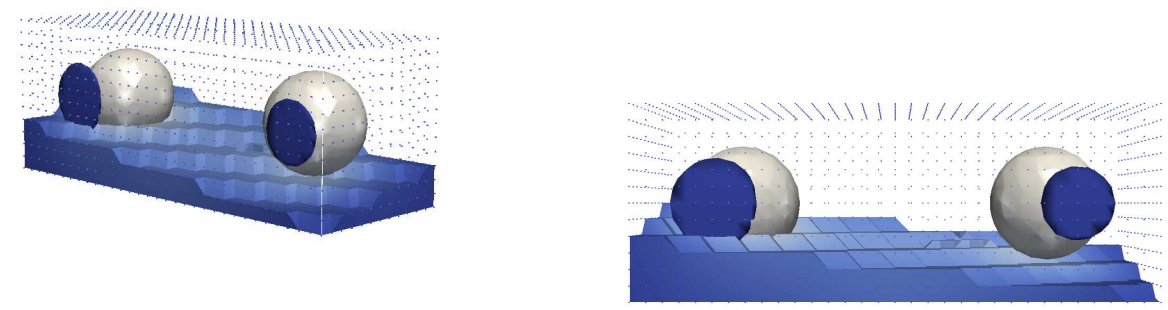

prospect view

$x_{1} x_{2}$ view
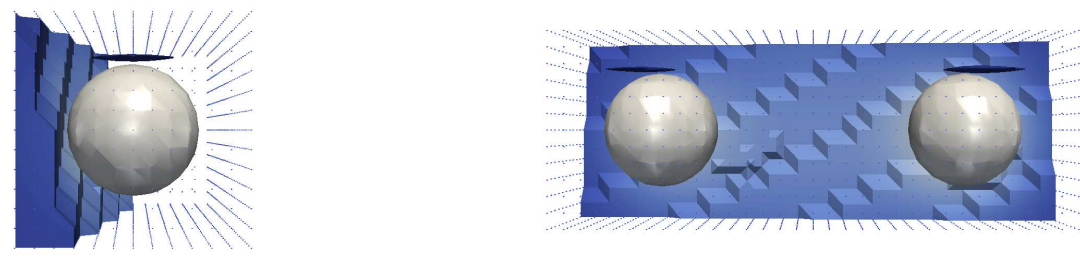

.

$\therefore$

$x_{2} x_{3}$ view

$x_{3} x_{1}$ view

Fig. 7 Test 2. Isosurfaces of the reconstructed function $c(x)$ with $\max _{\Omega_{F E M}} c(x)=5.58$ for $\omega=$ 40 in (2). Here, the noise level in data is $\sigma=10 \%$. We outline the spherical wireframe of the isosurface with exact value of the function (4), which corresponds to the value of the reconstructed $c=0.7 \max _{\Omega_{F E M}} c(x)$. 
Our numerical simulations show possibility of the reconstruction of function $c(x)$ in a hyperbolic problem (1) using a hybrid finite element/difference method of [5]. In our numerical tests, we have obtained stable reconstruction of the location and contrast of the function $c(x)$ in $x_{1} x_{2}$-directions for noisy backscattered data. The size and shape on $x_{3}$ direction should still be improved, and this can be done using, for example, an adaptive finite element method, see details in $[3,4,6]$.

\section{Acknowledgments}

The part of the research was done during the sabbatical stay of LB at the Institut de Mathématiques de Marseille, Aix-Marseille University, France, which was supported by the sabbatical programme at the Faculty of Science, University of Gothenburg, Sweden.

\section{References}

1. A. Bakushinsky, M. Y. Kokurin, A. Smirnova, Iterative Methods for Ill-posed Problems, Inverse and Ill-Posed Problems Series 54, De Gruyter, 2011.

2. L. Beilina, M. Cristofol and S. Li, Determining the conductivity for a non-autonomous hyperbolic operator in a cylindrical domain, Mathematical Methods in the Applied Sciences, to appear, 2018.

3. L. Beilina, M. Cristofol, S. Li and M. Yamamoto, Lipschitz stability for an inverse hyperbolic problem of determining two coefficients by a finite number of observations, Inverse problems, 34 015001, 2018.

4. L. Beilina, Adaptive hybrid FEM/FDM methods for inverse scattering problems. Inverse Problems and Information Technologies, 1(3), 73-116, 2002.

5. L. Beilina, Domain Decomposition finite element/finite difference method for the conductivity reconstruction in a hyperbolic equation, Communications in Nonlinear Science and Numerical Simulation, Elsevier, 2016, doi:10.1016/j.cnsns.2016.01.016

6. L. Beilina, N. T. Thành, M. V. Klibanov, J. Bondestam-Malmberg, Reconstruction of shapes and refractive indices from blind backscattering experimental data using the adaptivity, Inverse Problems, 30, 105007, 2014.

7. M. Bellassoued, Global logarithmic stability in inverse hyperbolic problem by arbitrary boundary observation, Inverse Problems, 20, 1033-1052, 2004.

8. M. Bellassoued, Uniqueness and stability in determining the speed of propagation of second-order hyperbolic equation with variable coefficients, Applicable Analysis, 83, 9831014, 2004.

9. M. Bellassoued, D. Jellali and M. Yamamoto, Lipschitz stability in in an inverse problem for a hyperbolic equation with a finite set of boundary data, Applicable Analysis 87, 1105-1119, 2008.

10. M. Bellassoued and M. Yamamoto, Logarithmic stability in determination of a coefficient in an acoustic equation by arbitrary boundary observation, J. Math. Pures Appl. 85, 193-224, 2006.

11. M. Bellassoued and M. Yamamoto, Determination of a coefficient in the wave equation with a single measurement, Applicable Analysis 87, 901-920, 2008. 
12. M. Cristofol, S. Li and E. Soccorsi, Determining the waveguide conductivity in a hyperbolic equation from a single measurement on the lateral boundary, J. Mathematical control and related fields $\mathbf{6},(3), 2016$.

13. R. Courant, K. Friedrichs and H. Lewy On the partial differential equations of mathematical physics, IBM Journal of Research and Development, 11(2), 215-234, 1967.

14. H. W. Engl, M. Hanke and A. Neubauer, Regularization of Inverse Problems (Boston: Kluwer Academic Publishers), 2000.

15. O. Imanuvilov and M. Yamamoto, Global Lipschitz stability in an inverse hyperbolic problem by interior observations, Inverse Problems 17, 717-728, 2001.

16. O. Imanuvilov and M. Yamamoto, Determination of a coefficient in an acoustic equation with single measurement, Inverse Problems 19, 157-171, 2003.

17. V. Isakov, Carleman type estimates and their applications, in New Analytic and Geometric Methods in Inverse Problems, K. Bingham, Y. Kurylev, and E. Somersalo, eds., SpringerVerlag, Berlin, 2004, pp. 93-125.

18. M. V. Klibanov, Inverse problems and Carleman estimates, Inverse Problems 8, 575-596, 1992.

19. M. V. Klibanov, Carleman estimates for global uniqueness, stability and numerical methods for coefficient inverse problems, J. Inverse Ill-Posed Probl. 21, 477-560, 2013.

20. M. V. Klibanov and M. Yamamoto, Lipschitz stability of an inverse problem for an accoustic equation, Applicable Analysis 85, 515-538, 2006.

21. S. Li and M. Yamamoto, An inverse problem for Maxwell's equations in isotropic and nonstationary media, Applicable Analysis 92 N 11, 2335-2356, 2013.

22. M. Yamamoto, Uniqueness and stability in multidimensional hyperbolic inverse problems, $\mathrm{J}$. Math. Pures Appl. 78, 65-98, 1999.

23. A. N. Tikhonov, A. V. Goncharsky, V. V. Stepanov and A. G. Yagola, "Numerical Methods for the Solution of Ill-Posed Problems" London: Kluwer, 1995. 